\title{
Computational Chemical Study of Pigment of Mangosteen (Garcinia mangostana) Rind Extract as Dye Compound in Dye-Sensitized Solar Cell (DSSC)
}

\author{
Didik Krisdiyanto $^{*, 1}$, Sudarlin $^{1}$, Hikmah Supriyati ${ }^{2}$ \\ ${ }^{1}$ Department of Chemistry, Faculty of Science and Technology, Universitas Islam Negeri Sunan Kalijaga Yogyakarta \\ Jl. Marsda Adisucipto No.1 Yogyakarta 55281, Tel. + 62-274-540971, Fax. + 62-274-519739, Indonesia \\ ${ }^{2}$ Post Graduate Program of Biological Education Department, Universitas Negeri Yogyakarta \\ Jalan Colombo No. 1 Yogyakarta 55281, Indonesia \\ Corresponding author* \\ didik_kris@yahoo.com
}

Manuscript received: 03 March, 2021. Revision accepted: 30 June, 2021. Published: 19 July, 2021.

\begin{abstract}
The electronic transition processes of $\alpha$-mangostin and $\beta$-mangostin as dye compounds in DSSC and their ability to transfer electrons to semiconductors have been studied in theory. The research was carried out computationally using the NwChem application. The methods used are Density Functional Theory (DFT) for structure optimization and Time-Dependent DFT (TD-DFT) for electronic transitions. The results showed that the energy of HOMO $\beta$-mangostin was lower that it was more stable or easier to form bonds with semiconductors. Likewise, its LUMO energy is lower than $\alpha$-mangostin that it is easier to inject electrons into the semiconductor. The energy difference of HOMO-LUMO $\beta$-mangostin is smaller than $\alpha$-mangostin. This shows that $\beta$-mangostin is more easily excited or more efficient in exciting solar energy to convert it into electricity. This is consistent with the molecular spectra where $\lambda_{\max } \beta$-mangostin is at a higher wavelength. Orbital analysis shows electron injection of $\alpha$-mangostin and $\beta$-mangostin into the semiconductor via double $\mathrm{O}$ atoms in each molecule. The injection is influenced by the bond length, where the bond length of $\alpha$-mangostin to $\mathrm{TiO} 2$ is smaller than that of $\beta$-mangostin to $\mathrm{TiO} 2$.
\end{abstract}

Keywords: $\alpha$-mangostin; $\beta$-mangostin; energy HOMO-LUMO; DFT; TD-DFT; mangosteen rind; Dye-Sensitized Solar Cell.

\section{INTRODUCTION}

Research on dye compounds for DSSC (Dye-Sensitized Solar Cell) has been widely carried out, but synthetic dyes' efficiency is still higher than natural dyes, which are only $\sim 1 \%$. However, synthetic dyes have drawbacks including difficult preparation, expensive production costs, and a toxic risk to the environment. The use of natural dyes becomes a very attractive alternative to develop because they are easy to obtain, have unlimited sources, have a long absorbance coefficient, high light capture efficiency, low production costs, easy preparation, and are environmentally friendly (Luo et al., 2009).

However, not all dyes can be used as sensitizers because they must qualify, namely adsorption intensity at visible wavelengths, strong adsorption on the semiconductor surface, has the ability to inject electrons into the semiconductor conduction band, and has groups $=\mathrm{O}$ or $-\mathrm{H}$ to bind with the $\mathrm{TiO} 2$ surface which can increase the rate of the electron transfer reaction (Ludin et. al., 2014).

Various studies on DSSC using natural dyes from plant extracts have been conducted and these studies have proven that natural dyes can provide a photovoltaic effect. One of the sensitizers used is mangosteen rind pigment extract. Chairat et al. (2007) in their research used mangosteen rind pigment extract which was proven to be used as a sensitizer on solar cells of the DSSC type. Mangosteen is very easy to obtain. This is because Indonesia is a tropical country that is very suitable for the growth of mangosteen.

The main component in the mangosteen pigment extract which is potential as a DSSC sensitizing dye is shown in Figure 1. The $\alpha$-mangostin and $\beta$-mangostin compounds are two of the several components in the mangosteen rind pigment extract which has absorption at visible wavelengths as one of the requirements for the DSSC dye. Further studies on the adsorption of compounds on semiconductor surfaces, electronic energy levels, and electron transfer capabilities need to be done. The study in this research was done with the help of computational chemistry. two basic structures of the main components of mangosteen rind extract, the electro-optical properties of DSSC will be studied based on computational calculations to study adsorption on semiconductors, adsorption spectrum, energy levels, and charge transfer. 


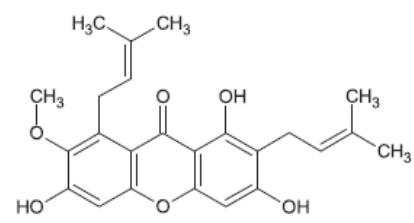

A

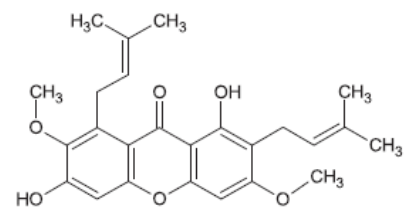

B
Figure 1. Basic structure of mangosteen rind extract (A) $\alpha$-mangostin (B) $\beta$-mangostin.

Dye-sensitized solar cell (DSSC) is a series of devices that can convert visible light into electricity based on semiconductor band gap sensitization and its bonding with groups on the surface of a thin layer cell (Ludin et. al., 2014). The DSSC material consists of a pair of glass substrates coated with TCO (Transparent Conducting Oxide) material which acts as an electrode and a counter electrode separated by a redox electrolyte which has high transparency and low resistance characteristics, can be Indium Tin Oxide (ITO), Aluminum Zinc Oxide (AZO), and Fluorine Tin Oxide (FTO) mounted opposite each other. FTO and ITO are most often used as DSSC, where the process of sintering the oxide layer on the substrate at a temperature of 450$500^{\circ} \mathrm{C}$, these materials have good conductivity and do not experience defects or defects in that temperature range (Halme, 2002).

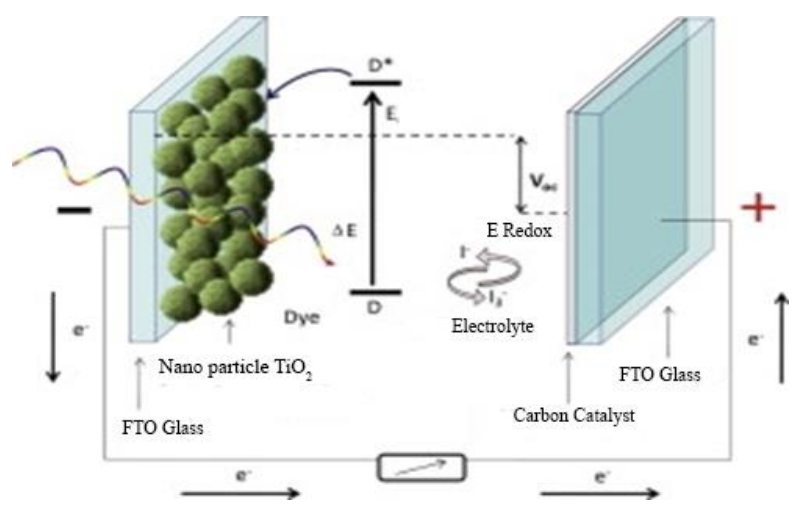

Figure 2. Schematic and working principle of Dye-Sensitized Solar Cell (DSSC).

The redox pair that is often used is I- / I3- (iodide / triiodide). The solvent used in the electrolyte solution is Polyethylene Glycol (PEG) because it can penetrate the $\mathrm{TiO} 2$ dye absorption for both small particle size comparisons and nano-scale pore diameters, and can maintain work stability (Misbahudin et.al., 2013). At TCO, the counter electrode is coated with a catalyst in the form of a carbon layer to accelerate the redox reaction, while the electrode is positioned as a porous $\mathrm{TiO} 2$ nanocrystalline layer as a photoanode and is sensitized by dye as a photosensitizer which functions to capture photons which then will be absorbed into $\mathrm{TiO} 2$ nanoparticles. An efficient photosensitizer must qualify, namely dye particles can be adsorbed onto the semiconductor surface, the capacity to capture light in the visible light range, the ability to inject electrons into the conduction band of the semiconductor, and have an $=\mathrm{O}$ or $-\mathrm{H}$ group to bind to the $\mathrm{TiO} 2$ surface. The working principle of the DSSC is shown in Figure 2.

Basically, the working principle of DSSC is a reaction of electron transfer where the first process begins with the excitation of electrons in the dye molecule due to photon absorption. Electrons are excited from the ground state (D) to excited state (E).

$\mathrm{S}+$ photon $\rightarrow \mathrm{S} *$ (Absorption)

The electrons from the excited state are then directly injected into the conduction band (Ecb) of the titania so that the dye molecules are oxidized $(\mathrm{S}+)$. With the presence of an electron donor by the electrolyte, the dye molecules return to their ground state and prevent the recapture of the oxidized dye electrons.

$\mathrm{S}^{*}+\mathrm{TiO} 2 \rightarrow \mathrm{e}-(\mathrm{TiO} 2)+\mathrm{S}+($ Injection process $)$

The injected electrons are carried from the $\mathrm{TiO} 2$ nanopore thin layer to the conductive electrode (anode).

e- ( $\mathrm{TiO} 2)+$ the conductive electrode $\rightarrow \mathrm{TiO} 2+\mathrm{e}-$ (c.e)

After reaching the TCO electrode, electrons flow towards the electrode counter through the external circuit and approach the cathode (counter electrode). The electrons are transferred to the electrolyte at the electrodes. With a catalyst on the counter electrode, the oxidized dye accepts electrons from the ion (I-), a redox reaction occurs to replace the lost electrons, and the iodide molecule is oxidized to the triiodide ion (I3-).

$\mathrm{S}++\frac{3}{2} \mathrm{I}-\rightarrow \mathrm{S}+\frac{1}{2} \mathrm{I} 3-$

Ion (I3-) is used to donate electrons to the oxidized dye permitting an electron transport cycle to be formed, with this cycle a direct conversion from sunlight to electricity.

$\frac{1}{2} \mathrm{I} 3-+$ e- (c.e) $\rightarrow \frac{3}{2} \mathrm{I}-+$ c.e

(Narayan, 2012).

Sunlight produces $45 \%$ of the spectra in the visible and $5 \%$ of the UV spectra so that in the use of dyes in the DSSC, the absorption of photon energy from visible light is carried out by light-sensitive materials (dyes that function as sensitizers). With the presence of a sensitizer, it is possible to inject/transfer electrons to the $\mathrm{TiO} 2$ semiconductor material even though the received photon energy is smaller than the band gap of the TiO2 semiconductor (this event is called sensitisation). To 
support this process, the $\mathrm{TiO} 2$ semiconductor material must be able to entangle as many molecules of the color substance as possible so that more electrons can be accepted.

Some of the criteria that must qualify by $\mathrm{TiO} 2$ as a semiconductor in DSSC include the particle size being on the nanometer scale. This is necessary because with the particle size that is on the nanometer scale the surface area of the particles as a whole becomes larger so that more dye molecules are possible to absorb. In addition, $\mathrm{TiO} 2$ particles are also expected to have a porous (mesoporous) morphology, allowing the dye molecules enter between the pores and can be absorbed on the surface of the $\mathrm{TiO} 2$ particles, which implies that they will increase the amount of light absorbed (Zhang et.al., 2008).

In addition, the use of semiconductor oxides in photoelectrochemistry is due to its stability against corrosion photo and also its large energy band (3.2-3.8 $\mathrm{eV}$ ) which in DSSC is needed for semiconductor transparency in most of the sunlight spectrum. Although $\mathrm{TiO} 2$ is a material that is often used because the efficiency of DSSC using $\mathrm{TiO}_{2}$ is still unmatched, several materials can act as semiconductor oxides including $\mathrm{ZnO}, \mathrm{CdSe}, \mathrm{CdS}, \mathrm{WO} 3, \mathrm{Fe} 3 \mathrm{O} 3, \mathrm{SnO} 2$, $\mathrm{Nb} 2 \mathrm{O} 5$ and Ta2O5 (Halme, 2002).

The dye in the DSSC oxide layer serves to capture photons of light. Furthermore, these photons are absorbed into the $\mathrm{TiO} 2$ nanoparticle. The requirements for dye as a photosensitizer in DSSC are absorption in the visible light region or near the infrared region of the sunlight spectrum and bind to the $\mathrm{TiO} 2$ semiconductor (Cherepy et.al., 1997). Functional groups are needed to interact with $\mathrm{TiO} 2$ surfaces such as carboxylates or around other acid groups (Galopini, 2004). Several functional groups have the possibility to bind with $\mathrm{TiO} 2$. The best groups are metal oxides such as phosphonic acid followed by carboxylic acids and their derivatives such as chlorides, amides, esters or carboxylic salts (Galopini, 2004).

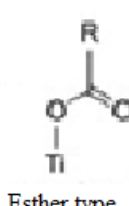

Esther type monodentate
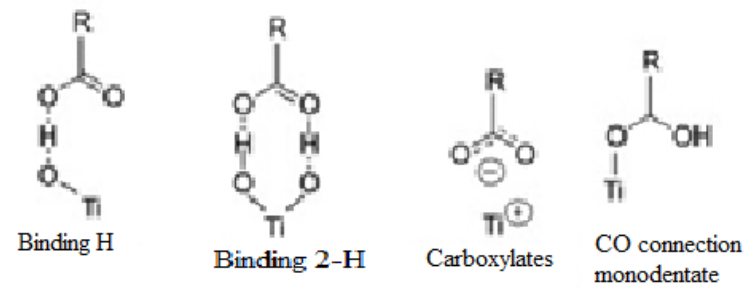

Figure 3. Possible bond models between $-\mathrm{COOH}$ groups and $\mathrm{TiO} 2$ (Hug et. al., 2014).
Plant extracts or pigments used as photosensitizers in visible light areas can be in the form of chlorophyll extract (Amoa, 2003). Chlorophyll is the main plant pigment which functions to absorb light and convert it into chemical energy needed to reduce carbon dioxide into carbohydrates in the photosynthesis process. Figure 3 shows several bonding models between $\mathrm{TiO} 2$ and the dye molecule with at least one carboxylate group.

Another requirement for this material to be an active ingredient in solar cells is that the material must be able to become a transfer medium for electric charge carriers as a result of the absorbed photons (Supriyanto et. al., 2010).

To obtain a suitable dye as a sensitizer requires a long time and resources in research which is computational chemistry can be an alternative. By knowing in more detail the electro-optical properties of a dye, it can be used as a basis for the development of efficient dye alternatives. Density Fungtional Theory (DFT) and Time Dependent DFT (TD-DFT) methods can be used for large molecules with an accurate approach to calculate the UV spectrum of dyes as well as for complex systems between dyes and semiconductors.

\section{METHODS}

The research was conducted computationally using the NwChem application. The methods used are Density Functional Theory (DFT) for structure optimization and Time Dependent DFT (TD-DFT) for electronic transitions. All calculations use B3LYP functional and 6-31G * base set. Data visualization using ECCE and ChemCraft applications. In the study of dyestuffs for DSSC in computational chemistry involving large molecules and complex systems, a suitable approach is needed.

\section{RESULTS AND DISCUSSION}

\section{Method Selection}

The calculation results obtained were compared with the results of experiments conducted by Madihah et al. (2012). The parameter being compared is the $\alpha$ mangostin excitation energy in units of $\mathrm{eV}$ as shown in Table 1. Other parameters such as bond length cannot be compared because experimental data are not available.

The results of the calculations as shown in Table 1 show the difference in excitation energy from the calculation of $\alpha$-mangostin and the experimental results of $0.01 \mathrm{eV}$. The similarity between the calculation results and the experimental results reached $99.71 \%$. These results indicate the selection of methods and basis sets in this study are correct and quite accurate. 
Table 1. Comparison of the first excitation energy of $\alpha$-mangostin on experimental and calculated results.

\begin{tabular}{llll}
\hline No & $\boldsymbol{\alpha}$-Mangostin Data & $\begin{array}{l}\text { Excitation energy } \\
(\mathbf{e V})\end{array}$ & $\begin{array}{l}\text { Similarity } \\
\text { level }\end{array}$ \\
\hline 1. & Experiment & 3.522 & $99,71 \%$ \\
2. & Calculation & 3.532 & \\
\hline
\end{tabular}

Supporting data that can be compared is the display of $\alpha$-mangostin spectra on the experimental and the calculation results at wavelengths above $280 \mathrm{~nm}$, which shows the similarities as shown in Figure 4.

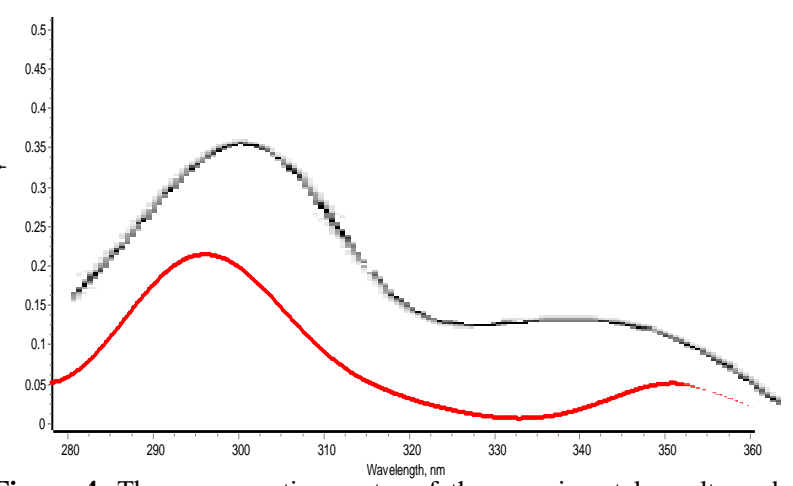

Figure 4. The $\alpha$-mangostin spectra of the experimental results and the calculation results above $280 \mathrm{~nm}$.

\section{Optimization of HOMO-LUMO molecules and energy}

Optimization is carried out on each molecule using the same method. The optimization results are in the form of a stable structure as shown in Figure 5 and the multivariate load value as shown in Table 2.

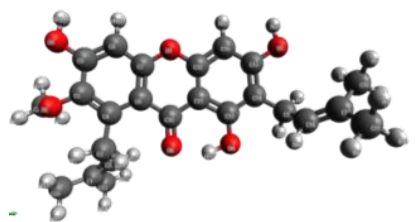

A

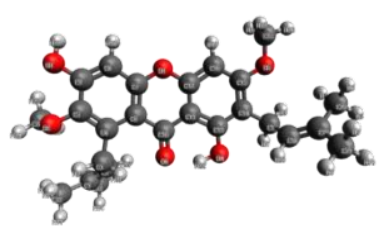

B
Figure 5. The results of the calculation of $\alpha$-mangostin (A) and $\beta$ mangostin (B).

Based on the optimization results of $\alpha$-mangostin and $\beta$-mangostin in Figure 5, each red $\mathrm{O}$ atom has a different mullicen charge value as shown in Table 2. Based on the table, the O-4 atom has a more negative charge so it is more reactive in binding to other atoms. Thus, O-4 atoms can be used to bind $\mathrm{TiO} 2$.

The HOMO and LUMO energies of each compound as a result of the optimization can be seen in Table 3 . Based on the HOMO energy in the table, $\beta$-mangostin has a lower HOMO energy. based on the requirements of the dye compound, this indicates that $\beta$-mangostin is more stable so it is easier to form bonds with semiconductors. Likewise, the LUMO $\beta$-mangostin energy is lower than the LUMO $\alpha$-mangostin energy so that $\beta$-mangostin is easier to inject electrons into the semiconductor.

Table 2. Mullicen charge values of $O \alpha$-mangostin and $\beta$-mangostin atoms.

\begin{tabular}{lll}
\hline Atom O & $\boldsymbol{\alpha}$-mangostin & $\boldsymbol{\beta}$-mangostin \\
\hline O-1 & $-0,135014$ & $-0,135076$ \\
O-2 & $-0,240754$ & $-0,240796$ \\
O-3 & $-0,360894$ & $-0,361235$ \\
O-4 & $-0,377779$ & $-0,380988$ \\
O-5 & $-0,370443$ & $-0,200601$ \\
O-6 & $-0,341725$ & $-0,341406$ \\
\hline
\end{tabular}

Table 3. The energy difference between HOMO-LUMO $\alpha$-mangostin and $\beta$-mangostin.

\begin{tabular}{lll}
\hline Energy & $\boldsymbol{\alpha}$-mangostin & $\boldsymbol{\beta}$-mangostin \\
\hline HOMO $(\mathrm{eV})$ & -5.647 & -5.677 \\
LUMO $(\mathrm{eV})$ & -1.547 & -1.783 \\
$\Delta(\mathrm{eV})$ & 4.100 & 3.894 \\
\hline
\end{tabular}

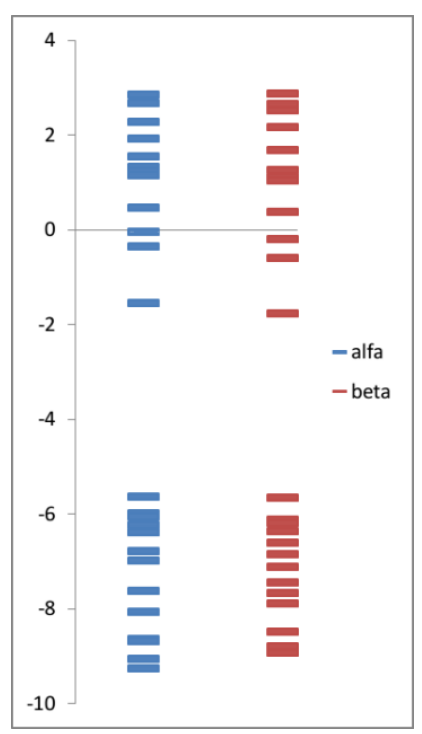

Figure 6. The energy bands of $\alpha$-mangostin and $\beta$-mangostin calculated.

The difference in energy bands produced is the difference between HOMO energy and LUMO energy. The smaller the value of the energy band difference, indicating that the easier the electrons in the molecule move from a lower energy level to a higher energy level. Figure 6 shows that the smaller HOMO-LUMO energy difference is $\beta$-mangostin. This shows that $\beta$-mangostin is more easily excited. That is, $\beta$-mangostin is more efficient in exciting solar energy to convert it into electricity.

The shape of the electron orbitals in the HOMO and LUMO states

The shape of the HOMO $\alpha$-mangostin and $\beta$-mangostin orbitals as presented in Figure 7 shows the same pattern. 
The figure shows the delocalized HOMO orbital electrons on the O-4 atom. This corresponds to the high mullicen charge of $\mathrm{O}-4$ atoms, thus strengthening the reason that $\mathrm{TiO} 2$ semiconductors tend to bond to $\mathrm{O}-4$ atoms, both to $\alpha$-mangostin and $\beta$-mangostin.

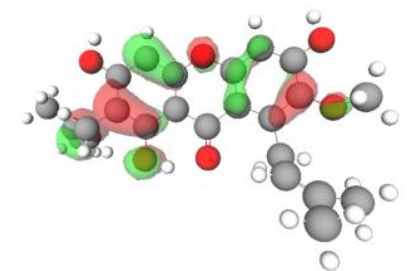

A

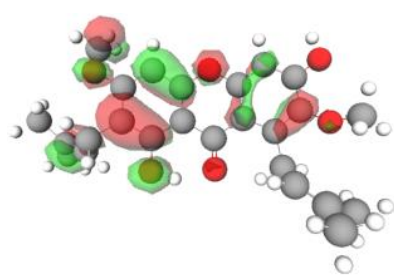

Figure 7. Form of the calculated $\alpha$-mangostin (A) and $\beta$-mangostin HOMO orbitals (B).

As with the HOMO orbitals, the LUMO $\alpha$-mangostin and $\beta$-mangostin orbitals show the same pattern as shown in Figure 8. The figure shows the delocalized molecular electrons on the O-6 atom. This shows the electron injection of the dye compound into the semiconductor via O-6 atoms, both in $\alpha$-mangostin and $\beta$-mangostin.

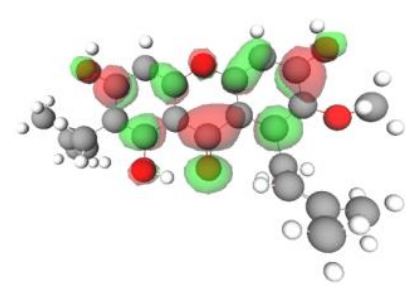

A

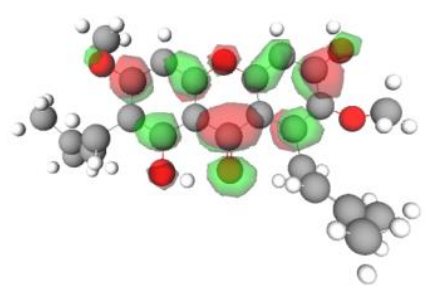

Figure 8. Form of the calculated LUMO $\alpha$-mangostin (A) and $\beta$ mangostin (B) orbitals.

\section{Bond Length}

The calculation of the bond length is carried out between $\mathrm{Ti}$ on $\mathrm{TiO} 2$ and $\mathrm{O}-6$ atoms in $\alpha$-mangostin and $\beta$ mangostin. In the previous section, it was explained that electrons are more easily injected into semiconductors via $\mathrm{O}-6$ atoms based on the electron composition of the LUMO orbitals. Thus, the distance between $\mathrm{Ti}$ and O- 6 atoms at $\alpha$ and $\beta$ mangostin will affect the electron transfer rate. The closer distance makes the electron transfer easier.

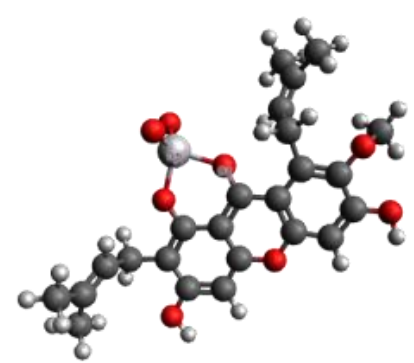

A

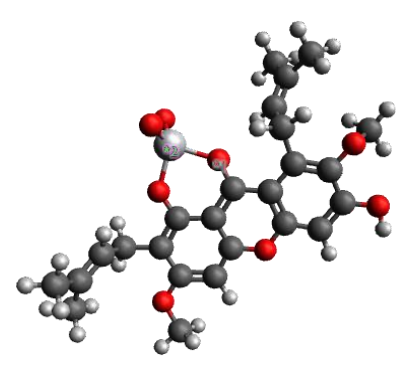

B
Figure 9. Optimization results for $\alpha$-mangostin $+\mathrm{TiO} 2$ (left) and $\beta$ mangostin $+\mathrm{TiO} 2$ (right).
The calculation results as shown in Table 4 show the length of each bond. The bond lengths between $\mathrm{Ti}$ and O-6 in $\alpha$-mangostin and $\beta$-mangostin are $1.989 \AA$ and $1.990 \AA$. When compared with the total radius length between $\mathrm{Ti}$ and $\mathrm{O}$ of $2.36 \AA$, it can be concluded that there is a bond between $\mathrm{TiO} 2$ and $\alpha$-mangostin and $\mathrm{TiO} 2$ and $\beta$-mangostin. Comparison of the bond length between Ti and O-6 in $\alpha$-mangostin and $\beta$-mangostin as in the table shows that $\alpha$-mangostin tends to transfer electrons more quickly to the $\mathrm{TiO} 2$ semiconductor.

Table 4. Bond lengths of each compound.

\begin{tabular}{|c|c|c|}
\hline Compound & Bond & Length $(\AA ̊)$ \\
\hline$\alpha$-mangostin & $\mathrm{Ti}-\mathrm{O}(6)$ & 1,989 \\
\hline$\beta$-mangostin & $\mathrm{Ti}-\mathrm{O}(6)$ & 1,990 \\
\hline
\end{tabular}

\section{Spectra}

The spectra in the figure show that the $\beta$-mangostin spectra have undergone a bathochromic shift. This is the effect of the addition of the $\mathrm{CH} 3$ group as an electron booster that is able to shift the wavelength to a higher wavelength. The shift in wavelength to Vis shows the efficiency of $\beta$-mangostin as a dye compound is better than $\alpha$-mangostin. This is because excitation at visible light (Vis) to infrared wavelengths is generally an excitation that involves the transfer of charge. Excitation involving charge transfer is an excitation that provides an effective injection of electrons into the semiconductor and thus plays a major role in determining the efficiency of DSSC cells.

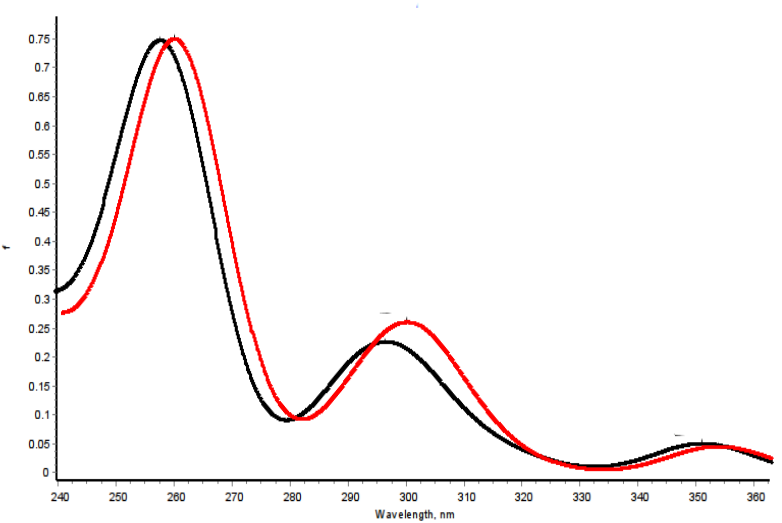

Figure 10. The calculated spectra of $\alpha$-mangostin (black curve) and $\beta$ mangostin (red curve).

In addition, the amount of sunlight reaching the earth's surface is dominated by UV rays at a wavelength of $300-400 \mathrm{~nm}$, which is about $95 \%$. UV light at 200300 wavelengths only reaches $5 \%$ of the earth's surface because most of it has been absorbed by airborne molecules such as ozone. 


\section{CONCLUSION}

The results showed that the energy of HOMO $\beta$ mangostin was lower that it was more stable or easier to form bonds with semiconductors. Likewise, LUMO energy is lower than $\alpha$-mangostin so that it is easier to inject electrons into the semiconductor. The energy difference of HOMO-LUMO $\beta$-mangostin is smaller than $\alpha$-mangostin. This shows that $\beta$-mangostin is more easily excited or more efficient in exciting solar energy to be converted into electricity.

Acknowledgments: LPPM UIN Sunan Kalijaga for funding assistance for this research in the 2016 beginner research program.

Conflict of interest: The authors declares that there are no conflicts of interest concerning the publication of this article.

\section{REFERENCES}

Amao, Yutaka \& Yamada, Yuriko \& Aoki, Keiko. (2004). Preparation and properties of dye-sensitized solar cell using chlorophyll derivative immobilized $\mathrm{TiO} 2$ film electrode. Journal of Photochemistry and Photobiology A: Chemistry. 164. 47-51. https://doi.org/ 10.1016/j.jphotochem.2003.11.011.

Chairat, M., Bremner, J. B., \& Chantrapromma, K., (2007). Dyeing of cotton and silk yarn with the extracted dye from the fruit hulls of mangosteen, Garcinia mangostana linn, Fibers and Polymers, 8(6); 613-619. https://doi.org/10.1007/BF02875998

Cherepy, N.J.; smestad, G.P.; Gratzel, M.; Zhang, J.Z. (1997). Ultrafast Electron Injection: Implications for a Photoelectrochemical Cell Utilizing on Anthocyanin DyeSensitized $\mathrm{TiO}_{2}$ Nanocrystalin electron. J. Phys Chem. 101: 9342-9351. https://doi.org/10.1021/jp972197w
Galopini, E. (2004). Linkers for Anchoring Sensitizers for Semiconductor Nanoparticels Coold. Chem Rev. 248: 12831297

Halme, E.J. (2002). Dye Sensitized Nanostructure and Organic Photovoltaic Cells: Technical Review and Pleminary Tests. Thesis. Departement of Engineering Physics and Mathematics Helsinki University of Technology. Espoo.

Hug, Hubert.; Bader, M; Mail, P.; Glatzer, T. (2014) Biophotovoltaics: Natural Pigments in Dye-Sensitized Solar Cells. Aplied Energy. 115, 216-225. https://doi.org/10.1016/j.apenergy.2013.10.055

Luo, P., Niu, H., Zheng, G., Bai, X., Zhang, M., \& Wang, W. (2009). From salmon pink to blue natural sensitizers for solar cells: Canna indica L., Salvia splendens, cowberry and Solanum nigrum L. Spectrochimica acta. Part A, Molecular and biomolecular spectroscopy, 74(4), 936-942. https://doi.org/10.1016/j.saa.2009.08.039

Madihah A.; Bohari M. Y.; Azwan M.L. (2012). Preliminary Study on Dispersion of $\alpha$-Mangostin in Pnipam Microgel System. The Malaysian Journal of Analytical Sciences, 16(3). $256-261$.

Misbachuddin, M.C.; Trihandaru; Suryasatria, Sutresno, A. (2013). Pembuatan prototipe dye sensitized solar cell (DSSC) dengan memanfaatkan ekstrak antosianin stawberry. Prosidingseminar nasional sains dan pendidikan sains fakultas MIPA UKSW.

Narayan, M. R. (2012). Review: Dye Sensitized Solar Cells Based On Natural Photosensitizers. Renewable And Sunstainable Energy. 16(1), 208-215. https://doi.org/10.1016/j.rser.2011.07.148

Supriyanto, Agus.; Kusminarto.; Triyana, K.; Roto.; Salleh, M.M.; Umar, A. A. (2010). Photosensitizing Effect of Porphyrin Films as Organic Photodetector. Journal of Materials Science and Engineering. 04: 40-44.

Zhang, D.; Lanier, S.M.; Downing, J.A.; Avent, J.L.; Lum, J. Jeanne, L. McHale. (2008). Betalain Pigments For dyesensitized solar cells. photochemistry and photobiology. 7280. 